\title{
AN INVERSE PROBLEM FOR A NONLINEAR SCHRÖDINGER EQUATION
}

\author{
BUI AN TON
}

Received 10 January 2002

We study the dependence on the control $q$ of the interval of definition of the solution $u$ of the Cauchy problem $u u^{\prime}+\Delta u=-\lambda|u|^{2} u-\imath q u$ in $\mathbb{R}^{2} \times(0, T), u(x, 0)=$ $\omega$ in $\mathbb{R}^{2}$, and we prove a version of Fibich's conjecture. Feedback laws for an inverse problem of the above equation with experimental data, measured on a portion of the boundary of an open, bounded subset of $\mathbb{R}^{2}$ are established.

\section{Introduction}

In this paper, we consider the Cauchy problem

$$
\begin{gathered}
\imath u^{\prime}+\Delta u=-\lambda|u|^{2} u-\imath q u \quad \text { in } \mathbb{R}^{2} \times(\tau, T), \\
u(x, \tau)=\omega=\omega_{1}+\imath \omega_{2} \quad \text { in } \mathbb{R}^{2}, \\
q(x)=q_{j}(x)+\imath q_{2}(x) ; \quad q_{j} \in H^{2}\left(\mathbb{R}^{2}\right), \lambda>0 .
\end{gathered}
$$

The existence of a local (in time) solution of (1.1) has been established by Ginibre and Velo [4], Kato [5], and others.

For positive constant $q,(1.1)$ may be considered as a model equation for the propagation of an intense laser beam through a medium with Kerr nonlinearity. The square of the transverse width of the incoming laser beam is proportional to $q$. Extensive investigations on the formation of the singularities of (1.1), with positive constant $q$, have been made by Fibich [3], Landman et al. [6], Landman et al. [7], LeMesurier et al. [8,9], and others.

In this paper, we consider $q$ as a control and we study the following:

(1) the dependence of the interval of definition of $u$ on $q$. It will be shown that there exists a threshold value $q_{\text {thres }} \in H^{2}\left(\mathbb{R}^{2}\right)$ such that

(i) the interval of definition $\left(\tau, T_{q}\right)$ increases with $\|q\|_{H^{2}\left(\mathbb{R}^{2}\right)}$ for $\|q\|_{H^{2}\left(\mathbb{R}^{2}\right)}$ $<\left\|q_{\text {thres }}\right\|_{H^{2}\left(\mathbb{R}^{2}\right)}$; 
(ii) the interval $\left(\tau, T_{q}\right)$ remains constant for all $q$ with $\|q\|_{H^{2}\left(\mathbb{R}^{2}\right)} \geq$ $\left\|q_{\text {thres }}\right\|_{H^{2}\left(\mathbb{R}^{2}\right)}$

(2) an inverse problem for (1.1). We wish to find $q$ and hence $u$, given the observed values of $u$ on a portion of the boundary of an open, bounded subset of $\mathbb{R}^{2}$.

The inverse problem for the linear Schrödinger equation in a bounded domain, namely finding the potential $q$ from the given spectra data, was solved by the celebrated Gelfand, Levitan, and Marchenko method. Recently, Advdonin et al. [1] introduced a new approach: finding the real-valued $q$ from the observed values of $u$ on a portion of the boundary of an open, bounded set. They applied the boundary control method and the exact controllability technique. In [12], the author used feedback control techniques to determine the complex potential from the observed values of $u$ on a portion of the boundary of an open subset of $\mathbb{R}^{n}$. It is the purpose of this paper to extend the method used in [12] to the case of the whole space. It is known that to get feedback laws, we must solve a nonlinear partial differential, involving the Clarke subgradients of the value function of the associated problem. As there are few information available on the Clarke subgradients and as there is no compact injection mapping in $\mathbb{R}^{2}$, difficulties arise when we wish to establish the weak continuity in some appropriate spaces of the Clarke subgradients. In this paper, we circumvent the problem by using an extension operator $\mathscr{E}$ of $H^{2}(G)$ into $H^{2}\left(\mathbb{R}^{2}\right)$.

Pioneering works on feedback laws for distributed systems of nonlinear parabolic equations, were done by Popa $[10,11]$ using a Trotter-type formula and the Clarke-Vinter optimization problem. Necessary conditions for the optimality were derived earlier by Barbu [2]. It turns out that by a suitable modification of the laws given by Popa, we can treat a wide range of problems with interacting state and control variables.

In Section 2, we give the notations, some known results, and we prove the Fibich conjecture. We study the value function in Section 3 and feedback laws are established in Section 4.

\section{Notations Fibich's conjecture}

Throughout the paper, $H$ is the Hilbert space $L^{2}\left(\mathbb{R}^{2}\right)$ with inner product $(\cdot, \cdot)$, norm $\|\cdot\|$, and $H^{k}$ is the usual Sobolev space $H^{k}\left(\mathbb{R}^{2}\right)$. From the Sobolev imbedding theorem, we deduce that $H^{2}$ is an algebra with respect to pointwise multiplication. We may rewrite (1.1) as

$$
\begin{gathered}
u_{1}^{\prime}+\Delta u_{2}+\lambda|u|^{2} u_{2}=q_{2} u_{2}-q_{1} u_{1} \quad \text { in } \mathbb{R}^{2} \times(\tau, T), \\
u_{2}^{\prime}-\Delta u_{1}-\lambda|u|^{2} u_{1}=-\left(q_{1} u_{2}+q_{2} u_{1}\right) \quad \text { in } \mathbb{R}^{2} \times(\tau, T), \\
u_{j}(x, \tau)=\omega_{j}(x) \quad \text { in } \mathbb{R}^{2} ; j=1,2 .
\end{gathered}
$$


Let

$$
\mathscr{2}_{j}=\left\{q_{j}:\left\|q_{j}\right\|_{H^{2}} \leq \alpha_{j}\right\}, \quad \alpha^{2}=\sum_{j=1}^{2} \alpha_{J}^{2}=1+\|\omega\|_{H^{2}}^{4} ; j=1,2 .
$$

Then $2_{j}$ is a closed convex subset of $H$.

Theorem 2.1. Let $\left\{\omega_{j}, q_{j}, \lambda, \sigma\right\}$ be in $H^{2} \times \mathscr{2}_{j} \times \mathbb{R}^{+} \times(0,1)$. Then there exist

(i) a nonempty interval $\left(\tau, T_{\lambda}(\alpha)\right)$,

(ii) a unique solution $u$ of (2.1) with

$$
\left\{u, u^{\prime}\right\} \in L^{\infty}\left(\tau, T_{\lambda}(\alpha) ; H^{2}\right) \times L^{\infty}\left(\tau, T_{\lambda}(\alpha) ; H\right)
$$

for $\|q\|_{H^{2}\left(\mathbb{R}^{2}\right)} \leq \alpha$. Furthermore, $T_{0}(\alpha)=T$ and there exists a constant $C$, independent of $\lambda$ and $q$ such that

$$
\begin{aligned}
& \|u\|_{L^{\infty}\left(\tau, T_{\lambda}(\alpha) ; H^{2}\right)}^{2}+\left\|u^{\prime}\right\|_{L^{\infty}\left(\tau, T_{\lambda}(\alpha) ; H\right)}^{2} \\
& \quad \leq C\|\omega\|_{H^{2}}^{2}\left\{1+\|q\|_{H^{2}}^{2}+\|\omega\|_{H^{2}}^{4}\right\} \exp \left(C \alpha^{2}(1-\sigma)^{-1} T_{\lambda}(\alpha)\right)
\end{aligned}
$$

for all solutions $u$ of (2.1) with $\|q\|_{H^{2}} \leq \alpha$.

Proof. The existence of a local (in time) solution of (2.1) is well known. We will establish the estimates and show the dependence of the solution on the control $q$.

(1) With (2.1), we have

$$
\frac{d}{d t}\|u(\cdot, t)\|^{2}+2 \lambda\|u(\cdot, t)\|_{L^{4}\left(\mathbb{R}^{2}\right)}^{4} \leq\|q\|_{L^{\infty}\left(\mathbb{R}^{2}\right)}\|u(\cdot, t)\|^{2} .
$$

It follows from the Gronwall lemma that

$$
\|u(\cdot, t)\|^{2}+\lambda\|u\|_{L^{4}\left(\tau, t ; L^{4}\left(\mathbb{R}^{2}\right)\right)}^{4} \leq C\|\omega\|^{2} \exp \left(C T\|q\|_{L^{\infty}\left(\mathbb{R}^{2}\right)}\right) .
$$

(2) We obtain, by differentiating (2.1) with respect to $t$

$$
\begin{aligned}
& u_{1}^{\prime \prime}+\Delta u_{2}^{\prime}+\lambda|u|^{2} u_{2}^{\prime}+2 \lambda\left(u_{1} u_{1}^{\prime}+u_{2} u_{2}^{\prime}\right) u_{2}=q_{2} u_{2}^{\prime}-q_{1} u_{1}^{\prime}, \\
& u_{2}^{\prime \prime}-\Delta u_{1}^{\prime}-\lambda|u|^{2} u_{1}^{\prime}-2 \lambda\left(u_{1} u_{1}^{\prime}+u_{2} u_{2}^{\prime}\right) u_{1}=-\left(q_{1} u_{2}^{\prime}+q_{2} u_{1}^{\prime}\right) .
\end{aligned}
$$

Taking the $H$-inner product of the first equation with $u_{1}^{\prime}$, and the second equation with $u_{2}^{\prime}$, we get

$$
\frac{d}{d t}\left\|u^{\prime}\right\|^{2} \leq C\left\{\|q\|_{H^{2}}\left\|u^{\prime}\right\|^{2}+\left\|u^{\prime}\right\|^{2}\|u\|_{H^{2}}^{2}\right\} .
$$

(3) Taking the $H$-inner product of the first equation of (2.1) with $\Delta u_{2}^{\prime}$, and the second equation with $-\Delta u_{1}^{\prime}$, we obtain

$$
\frac{d}{d t}\|\Delta u(\cdot, t)\|^{2} \leq \lambda C\left\|u^{\prime}\right\|_{H^{2}}^{3}+C\left\|u^{\prime}\right\|\|u\|_{H^{2}}
$$


Adding (2.6), (2.8), and (2.9), we get

$$
\begin{aligned}
\frac{d}{d t}\left\{\left\|u^{\prime}(\cdot, t)\right\|^{2}+\|u(\cdot, t)\|_{H^{2}}^{2}\right\} \leq & C\left\{\|q\|_{H^{2}}\left\|u^{\prime}(\cdot, t)\right\|^{2}+\|u(\cdot, t)\|^{2}\right\} \\
& +C \lambda^{2}\left\|u^{\prime}(\cdot, t)\right\|^{2}+\|u(\cdot, t)\|_{H^{2}}^{2} .
\end{aligned}
$$

We have applied the Hölder inequality in (2.10). It is clear that when $\lambda=0$, we get

$$
\begin{aligned}
\left\|u^{\prime}(\cdot, t)\right\|^{2}+\|u(\cdot, t)\|_{H^{2}}^{2} \leq & C\|\omega\|_{H^{2}}^{2}\left(1+\|q\|_{H^{2}}^{2}\right) \\
& +\left(1+\|q\|_{H^{2}}^{2}\right) \int_{\tau}^{t}\left\{\|u(\cdot, s)\|_{H^{2}}^{2}+\left\|u^{\prime}(\cdot, s)\right\|^{2}\right\} d s .
\end{aligned}
$$

The Gronwall lemma gives

$$
\left\|u^{\prime}\right\|_{L^{\infty}(\tau, T ; H)}^{2}+\|u\|_{L^{\infty}\left(\tau, T ; H^{2}\right)}^{2} \leq C\|\omega\|_{H^{2}}^{2}\left(1+\|q\|_{H^{2}}^{2}\right) \exp \left\{T\left(1+\|q\|_{H^{2}}^{2}\right)\right\} .
$$

Now we consider the case when $\lambda>0$. Set

$$
\varphi(t)=\|u(\cdot, t)\|_{H^{2}}^{2}+\left\|u^{\prime}(\cdot, t)\right\|^{2}
$$

then (2.10) may be rewritten as

$\frac{d}{d t}\left\{\varphi(t) \exp \left(-C t\|q\|_{H^{2}}\right)\right\} \leq C\left(1+\lambda^{2}\right) \exp \left(C t\|q\|_{H^{2}}\right)\left\{\varphi(t) \exp \left(-C t\|q\|_{H^{2}}\right)\right\}^{2}$.

It follows that

$$
\begin{aligned}
C \varphi(t)\left\{\|q\|_{H^{2}}+\lambda^{2} \varphi(\tau)[1\right. & \left.\left.-\exp \left(C(t-\tau)\|q\|_{H^{2}}\right)\right]\right\} \\
& \leq C \varphi(\tau)\|q\|_{H^{2}} \exp \left[C(t-\tau)\|q\|_{H^{2}}\right]
\end{aligned}
$$

for all $t \in\left[\tau, T_{\lambda}\left(\|q\|_{H^{2}}\right)\right]$ with

$$
T_{\lambda}\left(\|q\|_{H^{2}}\right)=\frac{1}{C\|q\|_{H^{2}}} \ln \left\{1+\frac{\sigma\|q\|_{H^{2}}}{C \lambda^{2}\|\omega\|_{H^{2}}^{2}\left(1+\|\omega\|_{H^{2}}^{4}+\|q\|_{H^{2}}^{2}\right)}\right\}, \quad \sigma \in(0,1) .
$$

We will write $T_{\lambda}(q)$ for $T_{\lambda}\left(\|q\|_{H^{2}}\right)$ when there is no possible confusion.

(4) Now we consider $T_{\lambda}(q)$, given by (2.16), and wish to find the maximum interval of definition $\left(\tau, T_{\lambda}(q)\right)$ of $u$ for all $q$ with $\|q\|_{H^{2}} \leq \alpha$ and for a fixed $\lambda>0$. We are led to the study of the function

$$
f(x)=\frac{1}{x} \ln \left(1+\frac{a x}{1+b+x^{2}}\right), \quad a, b, x>0 .
$$


A calculation gives

$$
f^{\prime}(x)=\left\{\frac{a x\left(1+b-x^{2}\right)}{\left(1+b+a x+x^{2}\right)\left(1+b+x^{2}\right)}-\ln \left(1+\frac{a x}{1+b+x^{2}}\right)\right\} x^{-2}=x^{-2} g(x) .
$$

Thus,

$$
f^{\prime}(x)<0 \quad \text { for } \alpha<x, \quad f(x)<f(\alpha) ; \quad \alpha^{2}=1+b=1+\|\omega\|_{H^{2}}^{4} .
$$

Since

$$
\inf \left\{f\left(\|q\|_{H^{2}}\right) \forall\|q\|_{H^{2}} \leq \alpha\right\}
$$

exists, we will take

$$
T_{\lambda}(\alpha)=\inf \left\{T ; \inf \left\{f\left(\|q\|_{H^{2}}\right) \forall\|q\|_{+H^{2}} \leq \alpha\right\}\right\} .
$$

(5) From (2.15), (2.16), (2.17), and (2.21), we deduce that

$$
\|u(\cdot, t)\|_{H^{2}}^{2}+\left\|u^{\prime}(\cdot, t)\right\|^{2} \leq C\|\omega\|_{H^{2}}^{2}\left(1+\alpha^{2}\right) \exp \left(C t \alpha^{2}\right)
$$

for all $t \in\left(\tau, T_{\lambda}(\alpha)\right)$ and for all $q$ with $\|q\|_{H^{2}} \leq \alpha$.

It is trivial to show that the solution is unique. The theorem is proved.

Thus for a given $q \in H^{2}$, there exists a unique solution of (2.1) on the interval $\left(\tau, T_{\lambda}(q)\right)$. We wish to find $\tilde{q} \in H^{2}$ with

$$
T_{\lambda}(\tilde{q})=\inf \left\{T ; \sup \left\{T_{\lambda}(q) \forall q,\|q\|_{H^{2}} \leq\|\tilde{q}\|_{H^{2}}\right\}\right\} .
$$

We are led to the study of Fibich's conjecture. Let $(\tau, T(\delta))$ be the interval of definition of (2.1) corresponding to the constant potential $q=\delta>0$. Fibich conjectures that $T(\delta)$ increases with $\delta$ up to a threshold value $\delta_{\text {thres }}$ and then $T(\delta)=T\left(\delta_{\text {thres }}\right)$ for $\delta \geq \delta_{\text {thres }}$.

Let $f(x)$ be as in (2.17) and let

$$
T(\beta)=\sup \{f(x): 0<x<\beta\} .
$$

From (2.15), it is clear that $(\tau, T(\beta))$ is the maximum interval of definition of the solution $u$ of (2.1) with $\|q\|_{H^{2}} \leq \beta$.

Theorem 2.2. Suppose that all the hypotheses of Theorem 2.1 are satisfied. Then there exists $q_{\text {thres }} \in H^{2}$ such that

(i) $T(\beta)<T(\gamma)$ for $\beta<\gamma<\left\|q_{\text {thres }}\right\|_{H^{2}}$,

(ii) $T\left(q_{\text {thres }}\right)=\sup \left\{f(x) \forall x, x \geq\left\|q_{\text {thres }}\right\|_{H^{2}}\right\}$.

Proof. With $f$ as in (2.17), we know that $f^{\prime}(x)<0$ for $x \geq \sqrt{1+b}$ where $b=$ $\|\omega\|_{H^{2}}^{4}$. So the function $f$ is decreasing for $x \geq \sqrt{1+b}$. 
Using a maximizing sequence, it is trivial to show that

$$
T_{\alpha}(x)=\sup \{f(\xi): 0<\xi \leq x \leq \alpha\}=f(\tilde{x}) \text { for some } \tilde{x} \in(0, x) .
$$

Set

$$
\mathscr{S}=\left\{\tilde{x}: f(\tilde{x})=T_{\alpha}(x), 0<x<\alpha\right\}
$$

Clearly $\mathscr{S}$ is nonempty and $\sup \mathscr{S}$ exists. Using a maximizing sequence, we obtain

$$
\sup \mathscr{S}=\hat{x} ; \quad f(\hat{x})=\sup \{f(\xi): 0<\xi \leq \alpha\}
$$

Let

$$
\tilde{T}_{\alpha}=f(\hat{x})
$$

then

$$
f(y)<f(\hat{x})=\tilde{T}_{\alpha}, \quad \forall y<\alpha .
$$

It follows from the definition of $T_{\alpha}(x)$ that

$$
T_{\alpha}(x)<T_{\alpha}(x), \quad \forall x, y \text { with } x<y<\alpha .
$$

In particular,

$$
T_{\hat{x}}(x)<T_{\hat{x}}(y), \quad \forall x, y \text { with } x<y<\hat{x} .
$$

Since $\hat{x} \leq \alpha$, we have from the definition of $\hat{x}$,

$$
f(y) \leq f(\hat{x}), \quad \forall y, \hat{x}<y \leq \alpha .
$$

On the other hand, $f$ is decreasing on $[\alpha, \infty)$ and thus

$$
f(y)<f(\alpha) \leq f(\hat{x}) .
$$

Combining inequalities (2.32) and (2.33), we get

$$
f(y)<f(\hat{x}), \quad \forall y, y>\hat{x} .
$$

Therefore,

$$
\sup \{f(y) \forall y>\hat{x}\}=f(\hat{x})=T_{\hat{x}} .
$$

From Theorem 2.1, we know that $\left(\tau, T_{\hat{x}}\right)$ is the maximum interval of definition of the solution $u$ of $(2.1)$ with potential $\|q\|_{H^{2}} \leq \hat{x}$. Thus $\hat{x}$ is the threshold value of the potential, as conjectured by Fibich. 
Let $G$ be a bounded open subset of $\mathbb{R}^{2}$ with a smooth boundary $\partial G$. It is known that there exists an extension mapping $\mathscr{E}$ of $H^{2}(G)$ into $H^{2}\left(\mathbb{R}^{2}\right)$ with

(i) $\mathscr{E} u(x)=u(x)$ a.e. in $G$;

(ii) $\|\mathscr{E} u\|_{H^{2}\left(\mathbb{R}^{2}\right)} \leq K\|u\|_{H^{2}(G)}$.

For a given $f \in H^{k}$, there exists a unique $v \in H^{k+2}$ such that

$$
v-\Delta v=f \quad \text { in } \mathbb{R}^{2} .
$$

Moreover, $\|v\|_{H^{2+k}} \leq C\|f\|_{H^{k}}$. The linear mapping $S$ of $H^{k}$ into $H^{k+2}$, defined by $S f=v$ where $v$ as in (2.36), is well defined and is bounded.

Let $\Gamma$ be a closed nonempty subset of $\partial G$ and let

$$
\chi=\chi_{1}+\chi_{\chi} ; \quad \chi_{j} \in L^{2}\left(0, T_{\lambda} ; L^{2}(\Gamma)\right), j=1,2
$$

be a function representing the measurement of the solution $u$ of (2.1) on $\Gamma$. With (2.1) we associate the cost function

$$
J(\tau ; u ; q ; \omega)=\sum_{j=1}^{2} \int_{\tau}^{T_{\lambda}} \int_{\Gamma}\left|\chi_{j}(\cdot, t)-u_{j}(\cdot, t)\right|^{2} d \sigma d t .
$$

The purpose of this paper is to find $\tilde{q} \in \mathcal{2}$ so that the solution $\tilde{u}$ of (2.1), with potential $q=\tilde{q}$, satisfies the relation

$$
V(\tau ; \omega)=J(\tau ; \tilde{u} ; \tilde{q} ; \omega)=\inf \{J(\tau ; u ; q ; \omega) \forall q \in 2\} .
$$

We are led to the study of the feedback laws for the optimization problem (2.39).

Clearly, when $\chi$ represents the experimental values of the solution $u$ of (2.1), corresponding to some potential $q \in H^{2}$, then the value function $V(\tau ; \omega)$ is equal to zero.

Let $k_{j}^{*}$ be the mapping of $L^{2}\left(\tau, T_{\lambda} ; H\right)$ into $\mathbb{R}$ defined by the equation

$$
k_{j}^{*}\left(v_{j}\right)=\sup \left\{\left(q_{j}, v_{j}\right)_{L^{2}\left(\tau, T_{\lambda} ; H\right)} \forall q_{j} \in 2_{j}\right\} ; \quad j=1,2 .
$$

Then $k_{j}^{*}$ is a lower semi-continuous (l.s.c.) convex mapping of $L^{2}\left(\tau, T_{\lambda} ; H\right)$ into $\mathbb{R}$. A trivial argument shows that there exists $q_{j}^{*} \in \mathscr{2}_{j}$ such that

$$
k_{j}^{*}\left(v_{j}\right)=\left(q_{j}^{*}, v_{j}\right)_{L^{2}\left(\tau, T_{\lambda} ; H\right)} .
$$

Since $k_{j}^{*}$ are l.s.c. convex mappings of $L^{2}\left(\tau, T_{\lambda} ; H\right)$ into $\mathbb{R}$, its subdifferentials exist and are mappings of $L^{2}\left(\tau, T_{\lambda} ; H\right)$ into $L^{2}\left(\tau, T_{\lambda} ; H\right)$. We have

$$
\begin{aligned}
k_{j}^{*}\left(w_{j}\right)-k_{j}^{*}\left(v_{j}\right) & \geq\left(p_{j}, w_{j}\right)_{L^{2}\left(\tau, T_{\lambda} ; H\right)}-\left(q_{j}^{*}, v_{j}\right)_{L^{2}\left(\tau, T_{\lambda} ; H\right)} \\
& \geq\left(q_{j}^{*}, w_{j}-v_{j}\right)_{L^{2}\left(\tau, T_{\lambda} ; H\right)}, \quad \forall p_{j} \in 2_{j} .
\end{aligned}
$$

Hence $q_{j}^{*} \in \partial k_{j}^{*}\left(v_{j}\right)$. 
392 An inverse problem for a nonlinear Schrödinger equation

\section{The value function}

In this section, we show that the value function of problem (2.1), (2.38), given by (2.39), has Clarke subgradients. The main results of this section are the following two theorems.

Theorem 3.1. Suppose that all the hypotheses of Theorem 2.1 are satisfied. Let $\chi$ be an element of $L^{2}\left(\tau, T_{\lambda} ; L^{2}(\Gamma)\right)$ and let $V(\tau ; \omega)$ be as in (2.39). Then there exists a positive constant $C$ such that

$$
|V(\tau ; \omega)-V(\tau ; \theta)| \leq C\|\omega-\theta\|_{H^{2}}, \quad \forall \omega, \theta \text { with }\|\omega\|_{H^{2}}+\|\theta\|_{H^{2}} \leq 1 .
$$

The Clarke subgradients $\partial_{1} V\left(\tau ; \omega_{1} ; \omega_{2}\right), \partial_{2} V\left(\tau ; \omega_{1} ; \omega_{2}\right)$ of $V$ with respect to $\omega_{1}$, $\omega_{2}$, respectively, exist and

$$
\left\|\partial_{1} V\right\|_{L^{2}\left(\tau, T_{\lambda} ; H^{-2}\right)}+\left\|\partial_{2} V\right\|_{L^{2}\left(\tau, T_{\lambda} ; H^{-2}\right)} \leq C .
$$

The generic constant $C$ is independent of both $\lambda$ and $\omega$.

The following theorem will be needed.

Theorem 3.2. Suppose that all the hypotheses of Theorem 3.1 are satisfied. Let $S$ be the bounded linear mapping of $H^{k}$ into $H^{k+2}$, given by (2.36), and let $\mathscr{E}$ be the extension mapping of $H^{2}(G)$ into $H^{2}\left(\mathbb{R}^{2}\right)$. Then

$$
|V(\tau ; S \mathscr{E}(\omega))-V(\tau ; S \mathscr{E}(\theta))| \leq C\|\omega-\theta\|_{L^{2}(G)}
$$

for all $\omega, \theta$ with $\|\omega\|+\|\theta\| \leq 1$.

The Clarke subgradients $\partial_{j} V(\tau ; S \mathscr{E}(\omega))$ of $V(\tau ; S \mathscr{E}(\omega))=\mathscr{V}(\tau ; \omega)$ with respect to $\omega_{j}$ exist and

$$
\left\|\partial_{j} V(\cdot, S \mathscr{E}(\omega))\right\|_{L^{2}\left(\tau, T_{\lambda} ; L^{2}(G)\right)} \leq C .
$$

The constant $C$ is independent of $\lambda$ and $\omega$.

We have the following lemma.

Lemma 3.3. Suppose that all the hypotheses of Theorem 3.1 are satisfied. Then there exists

$$
\left\{\tilde{u}, \tilde{u}^{\prime}, \tilde{q}\right\} \in L^{\infty}\left(\tau, T_{\lambda} ; H^{2}\right) \times L^{2}\left(\tau, T_{\lambda} ; H\right) \times 2
$$

with $\tilde{u}$ being the unique solution of (2.1), corresponding to the potential $\tilde{q}$ and such that

$$
V(\tau ; \omega)=J(\tau ; \tilde{u} ; \tilde{q} ; \omega)=\inf \{J(\tau ; u ; q ; \omega) \forall q \in 2\} .
$$

Proof. Let $\left\{u^{n}, q^{n}\right\}$ be a minimizing sequence with

$$
J\left(\tau ; u^{n} ; q^{n} ; \omega\right)-\frac{1}{n} \leq V(\tau ; \omega)
$$


Since $q^{n} \in 2$, there exists a subsequence, denoted again by $q^{n}$, such that $q^{n} \rightarrow$ $\tilde{q}$ in $H_{\text {weak }}^{2}$ with $\tilde{q} \in 2$. From the estimates of Theorem 2.1, we obtain, by taking subsequences,

$$
\left\{u^{n},\left(u^{n}\right)^{\prime}\right\} \longrightarrow\left\{\tilde{u}, \tilde{u}^{\prime}\right\} \text { in }\left(L^{\infty}\left(\tau, T_{\lambda} ; H^{2}\right)\right)_{\text {weak }^{*}} \times\left(L^{\infty}\left(\tau, T_{\lambda} ; H\right)\right)_{\text {weak }}{ }^{*} .
$$

From the 1.s.c. of $J$, we deduce that

$$
J(\tau ; \tilde{u} ; \tilde{q} ; \omega)=V(\tau ; \omega)
$$

The lemma is proved.

Proof of Theorem 3.1. (1) Let $\omega, \theta$ be in $H^{2}$, then

$$
\begin{aligned}
V(\tau ; \theta)-V(\tau ; \omega) & =V(\tau ; \theta)-J(\tau ; \tilde{u} ; \tilde{q} ; \omega) \\
& \leq J(\tau ; v ; \tilde{q} ; \theta)-J(\tau ; \tilde{u} ; \tilde{q} ; \omega) \\
& \leq \sum_{j=1}^{2}\left\|v_{j}-\tilde{u}_{j}\right\|_{L^{2}\left(\tau, T_{\lambda}: L^{2}(\Gamma)\right)}^{2},
\end{aligned}
$$

where $v$ is the solution of (2.1) with potential $\tilde{q}$, initial value $\theta$. Thus, we have

$$
\begin{gathered}
\imath(\tilde{u}-v)^{\prime}+\Delta(\tilde{u}-v)=-\lambda|\tilde{u}-v|^{2}(\tilde{u}-v)-\imath \tilde{q}(\tilde{u}-v), \\
\tilde{u}(\tau ; \cdot)-v(\tau ; \cdot)=\omega-\theta .
\end{gathered}
$$

It follows from Theorem 2.1 that

$$
\|\tilde{u}-v\|_{L^{\infty}\left(\tau, T_{\lambda} ; H^{2}\right)}+\left\|(\tilde{u}-v)^{\prime}\right\|_{L^{\infty}\left(\tau, T_{\lambda} ; H\right)} \leq C\|\theta-\omega\|_{H^{2}}
$$

Therefore,

$$
\begin{aligned}
\|\tilde{u}-v\|_{L^{2}\left(\tau, T_{\lambda} ; L^{2}(\Gamma)\right)} & \leq C\|\tilde{u}-v\|_{L^{2}\left(\tau, T_{\lambda} ; H^{1}(G)\right)} \\
& \leq C\|\tilde{u}-v\|_{L^{2}\left(\tau, T_{\lambda} ; H^{2}\right)} \\
& \leq C\|\omega-\theta\|_{H^{2}}
\end{aligned}
$$

We obtain

$$
V(\tau ; \theta)-V(\tau ; \omega) \leq C\|\omega-\theta\|_{H^{2}}
$$

for all $\|\omega\|_{H^{2}}+\|\theta\|_{H^{2}} \leq 1$.

Reversing the role of $\omega$ and $\theta$ we get

$$
V(\tau ; \omega)-V(\tau ; \theta) \leq C\|\omega-\theta\|_{H^{2}}
$$

for all $\|\omega\|_{H^{2}}+\|\theta\|_{H^{2}} \leq 1$. 
394 An inverse problem for a nonlinear Schrödinger equation

Combining (3.14) and (3.15), we obtain

$$
|V(\tau ; \omega)-V(\tau ; \theta)| \leq C\|\omega-\theta\|_{H^{2}}
$$

for all $\|\omega\|_{H^{2}}+\|\theta\|_{H^{2}} \leq 1$.

Taking $\theta=\left(\theta_{1}, \omega_{2}\right)$ in the above expression, we obtain

$$
\left|V\left(\tau ; \omega_{1} ; \omega_{2}\right)-V\left(\tau ; \theta_{1} ; \omega_{2}\right)\right| \leq C|| \omega_{1}-\theta_{1} \|_{H^{2}}
$$

for all $\left\|\theta_{1}\right\|_{H^{2}}+\left\|\omega_{1}\right\|_{H^{2}} \leq 1$.

Therefore $\partial_{1} V\left(\tau ; \cdot ; \omega_{2}\right)$, the Clarke subgradient of $V\left(\tau ; \cdot ; \omega_{2}\right)$ exists and

$$
\left\|\partial_{1} V\right\|_{L^{2}\left(\tau, T_{\lambda} ; H^{-2}\right)} \leq C
$$

Similarly for $\partial_{2} V\left(\tau ; \omega_{1} ; \cdot\right)$. Thus the theorem is proved.

Proof of Theorem 3.2. As in Theorem 3.1, we have

$$
\begin{aligned}
|V(\tau, S \mathscr{E}(\omega))-V(\tau ; S \mathscr{E}(\theta))| & \leq C\|S \mathscr{E}(\omega)-S \mathscr{E}(\theta)\|_{H^{2}} \\
& \leq C\|\mathscr{E}(\omega-\theta)\|_{H} \\
& \leq C\|\omega-\theta\|_{L^{2}(G)}
\end{aligned}
$$

for all $\|\theta\|_{L^{2}(G)}+\|\omega\|_{L^{2}(G)} \leq 1$.

We have made use of the properties of the extension operator $\mathscr{E}$ and of $S$. Therefore, the Clarke subgradients $\partial_{j} V(\tau ; S \mathscr{E}(\omega))$ of $\mathscr{V}(\tau ; \omega)=V(\tau ; S \mathscr{E}(\omega))$ with respect to $\omega_{j}$ exist and are, moreover, bounded mappings of $L^{2}\left(\tau, T_{\lambda} ; L^{2}(G)\right)$ into the closed convex subsets of $L^{2}\left(\tau ; T_{\lambda} ; L^{2}(G)\right)$. Furthermore,

$$
\left\|p_{j}\right\|_{L^{2}\left(\tau, T_{\lambda} ; L^{2}(G)\right)} \leq C, \quad \forall p_{j} \in \partial_{j} V(\tau ; S \mathscr{E}(\omega)) .
$$

The theorem is proved.

\section{Feedback laws}

Let $k_{j}^{*}$ be as in (2.40) and let $V$ be the value function associated with (2.1), (2.38) and defined by (2.39). Consider the initial value problem

$$
\begin{gathered}
\imath u^{\prime}+\Delta u=-\lambda|u|^{2} u-\imath \tilde{q} u \quad \text { in } \mathbb{R}^{2} \times\left(\tau, T_{\lambda}\right), \\
u(x, \tau)=\omega, \\
k_{j}^{*}\left(\mathscr{E}\left(p_{j}\right)\right)=\left(\mathscr{E}\left(p_{j}\right), \tilde{q}_{j}\right)_{L^{2}\left(\tau, T_{\lambda} ; H\right)}, \quad \mathscr{E}\left(p_{j}\right) \in \partial_{j} V\left(t ; S \mathscr{E}\left(\left.u\right|_{G}\right)\right), \\
\tilde{q}_{j} \in \partial k_{j}^{*}\left(\mathscr{E}\left[\partial_{j} V\left(t ; S \mathscr{E}\left(\left.u\right|_{G}\right)\right)\right]\right) ; \quad j=1,2 .
\end{gathered}
$$

Nonlinear problems of the type considered in (4.1), arise in the study of feedback laws of nonlinear-parabolic-equation distributed systems. Let

$$
\mathscr{B}_{\tilde{C}}=\left\{\left.v\right|_{G}:\|v\|_{L^{2}\left(\tau, T_{\lambda} ; H^{2}\right)}+\left\|v^{\prime}\right\|_{L^{2}\left(\tau, T_{\lambda} ; H\right)} \leq \tilde{C}\right\}
$$


with

$$
\tilde{C}=C\left(1+\alpha+\|\omega\|_{H^{2}}\right) \exp (C T \alpha)
$$

and $\alpha$ is as in Theorem 2.1.

Since $G$ is a bounded open subset of $\mathbb{R}^{2}$, it is clear that $\mathscr{B}_{\tilde{C}}$ is a compact convex subset of $L^{2}\left(\tau, T_{\lambda} ; L^{2}(G)\right)$.

Let $v \in \mathscr{B}_{\tilde{C}}$, then $\partial_{j} V\left(t ; S \mathscr{E}\left(\left.v\right|_{G}\right)\right)$ exists and maps $L^{2}\left(\tau, T_{\lambda} ; L^{2}(G)\right)$ into the closed convex subsets of $L^{2}\left(\tau, T_{\lambda} ; L^{2}(G)\right)$, with $\partial_{j} V\left(t ; S \mathscr{E}\left(\left.v\right|_{G}\right)\right)$, being closed convex subsets of $L^{2}\left(\tau, T_{\lambda} ; L^{2}(G)\right)$, there exists a unique element $p_{j}(v) \in$ $\partial_{j} V\left(t ; S \mathscr{E}\left(\left.v\right|_{G}\right)\right)$ of minimal $L^{2}\left(\tau, T_{\lambda} ; L^{2}(G)\right)$-norm.

By the same argument, there exists a unique element $\tilde{q}_{j} \in \partial k_{j}^{*}\left(S \mathscr{E}\left(p_{j}\right)\right)$ of the closed convex set

$$
k_{j}^{*}\left(\mathscr{E} p_{j}\right)=\left(\mathscr{E} p_{j}, q_{j}\right)_{L^{2}\left(\tau, T_{\lambda} ; H\right)}, \quad q_{j} \in \mathscr{2}_{j}
$$

with minimal $L^{2}\left(\tau, T_{\lambda} ; H\right)$-norm.

Consider the initial value problem

$$
\begin{aligned}
\imath u^{\prime}+\Delta u+\lambda|u|^{2} u & =-\imath \tilde{q}(v) u & & \text { in } \mathbb{R}^{2} \times\left(\tau, T_{\lambda}\right), \\
u(x, \tau) & =\omega & & \text { in } \mathbb{R}^{2} .
\end{aligned}
$$

For each $v \in \mathscr{B}_{\tilde{C}}$, there exists a unique solution $u$ of problem (4.5). Let $A$ be the nonlinear mapping of $\mathscr{T}_{\tilde{C}}$, considered as a subset of $L^{2}\left(\tau, T_{\lambda} ; L^{2}(G)\right)$ into $L^{2}\left(\tau, T_{\lambda} ; L^{2}(G)\right)$ defined by

$$
A(v)=\left.u\right|_{G}
$$

With the estimates of Theorem 2.1, we can check that $A$ maps $\mathscr{B}_{\tilde{C}}$ into itself. Now we show that $A$ satisfies all the hypotheses of the Schauder fixed point theorem.

THeorem 4.1. Suppose that all the hypotheses of Theorems 2.1 and 3.2 are satisfied. Then there exists a solution $\tilde{u}$ of (4.1) with

$$
\left\{\tilde{u}, \tilde{u}^{\prime}\right\} \in L^{\infty}\left(\tau, T_{\lambda} ; H^{2}\right) \times L^{\infty}\left(\tau, T_{\lambda} ; H\right)
$$

Proof. Let $v^{n}$ be in $\mathscr{B}_{\tilde{C}}$, then from Aubin's theorem there exists a subsequence such that $\left\{v^{n},\left(v^{n}\right)^{\prime}\right\} \rightarrow\left\{v, v^{\prime}\right\}$ in $\left(L^{\infty}\left(\tau, T_{\lambda} ; H^{2}(G)\right)\right)_{\text {weak }^{*}} \cap C\left(\tau, T_{\lambda} ; H^{1}(G)\right) \times$ $\left(L^{\infty}\left(\tau, T_{\lambda} ; H\right)\right)_{\text {weak }}$ and $v \in \mathscr{B}_{\tilde{C}}$. We have

$$
\begin{aligned}
\left\|S \mathscr{E}\left(v^{n}\right)-S \mathscr{E}(v)\right\|_{C\left(\tau, T_{\lambda} ; H^{3}\right)} & \leq C\left\|\mathscr{E}\left(v^{n}\right)-\mathscr{E}(v)\right\|_{C\left(\tau, T_{\lambda} ; H^{1}\right)} \\
& \leq C\left\|v^{n}-v\right\|_{C\left(\tau, T_{\lambda} ; H^{1}(G)\right) .}
\end{aligned}
$$

Hence $S \mathscr{E}\left(v^{n}\right) \rightarrow \mathscr{E}(v)$ in $C\left(\tau, T_{\lambda} ; H^{3}\right)$. 
Let $p_{j}\left(v^{n}\right)$ be the unique element of $\partial_{j} V\left(t ; S \mathscr{E}\left(\left.v^{n}\right|_{G}\right)\right)$ with minimal $L^{2}\left(\tau, T_{\lambda} ; L^{2}(G)\right)$-norm. From Theorem 3.2, we get

$$
\left\|p_{j}\left(v^{n}\right)\right\|_{L^{2}\left(\tau, T_{\lambda} ; L^{2}(G)\right)} \leq C .
$$

Thus there exists a subsequence such that

$$
p_{j}\left(v^{n}\right) \longrightarrow \hat{p}_{j} \quad \text { in }\left(L^{2}\left(\tau, T_{\lambda} ; L^{2}(G)\right)\right)_{\text {weak }} .
$$

Now we show that $\hat{p}_{j} \in \partial_{j} V\left(t ; S \mathscr{E}\left(\left.v\right|_{G}\right)\right)$ with minimal $L^{2}\left(\tau, T_{\lambda} ; L^{2}(G)\right)$-norm. From the definition of subgradients, we have

$$
\begin{aligned}
\int_{\tau}^{T_{\lambda}}\left\{V\left(t ; S \mathscr{E}\left(\left.w_{1}\right|_{G}\right), S \mathscr{E}\left(\left.v_{2}^{n}\right|_{G}\right)\right)\right. & \left.-V\left(t ; S \mathscr{E}\left(\left.v_{1}^{n}\right|_{G}\right)\right), S \mathscr{E}\left(\left.v_{2}^{n}\right|_{G}\right)\right\} d t \\
& \geq \int_{\tau}^{T_{\lambda}}\left(p_{1}\left(v^{n}\right), w_{1}-v_{1}^{n}\right)_{L^{2}(G)} d t .
\end{aligned}
$$

It follows from Theorem 3.2 that

$$
\begin{aligned}
\int_{\tau}^{T_{\lambda}}\left\{V\left(t ; S \mathscr{E}\left(\left.w_{1}\right|_{G}\right), S \mathscr{E}\left(\left.v_{2}\right|_{G}\right)\right)\right. & \left.-V\left(t ; S \mathscr{E}\left(\left.v_{1}\right|_{G}\right), S \mathscr{E}\left(\left.v_{2}\right|_{G}\right)\right)\right\} d t \\
& \geq \int_{\tau}^{T_{\lambda}}\left(\hat{p}_{1}, w_{1}-v_{1}\right)_{L^{2}(G)} d t
\end{aligned}
$$

for all $w_{1} \in L^{2}\left(\tau, T_{\lambda} ; L^{2}(G)\right)$. Thus,

$$
\hat{p}_{1} \in \partial_{1} V\left(t ; S \mathscr{E}\left(\left.v_{1}\right|_{G}, S \mathscr{E}\left(\left.v_{2}\right|_{G}\right)\right)\right) .
$$

Similarly for $\hat{p}_{2}$. Now it remains to show that $\hat{p}_{j}$ is the unique element of $\partial_{j} V\left(t ; S \mathscr{E}\left(\left.v\right|_{G}\right)\right)$ with minimum $L^{2}\left(\tau, T_{\lambda} ; L^{2}(G)\right)$-norm.

Let

$$
B_{\varepsilon}(v)=\left\{v^{\varepsilon}: v^{\varepsilon} \in \mathscr{B}_{\tilde{C}},\left\|v^{\varepsilon}-v\right\|_{L^{2}\left(\tau, T_{\lambda} ; H^{2}(G)\right)}+\left\|\left(v^{\varepsilon}\right)^{\prime}-v^{\prime}\right\|_{L^{2}\left(\tau, T_{\lambda} ; L^{2}(G)\right)} \leq \varepsilon\right\} .
$$

Then

$$
\bigcap_{\varepsilon}\left\{\partial_{j} V\left(t ; S \mathscr{E}\left(\left.v^{\varepsilon}\right|_{G}\right)\right): v^{\varepsilon} \in B_{\mathcal{\varepsilon}}(v)\right\} \subset \partial_{j} V\left(t ; S \mathscr{E}\left(\left.v^{n}\right|_{G}\right)\right)
$$

since $v^{n} \in B_{\varepsilon}(v)$ for $n \geq n_{0}$. Hence

$$
\left\|p_{j}\left(v^{n}\right)\right\|_{L^{2}\left(\tau, T_{\lambda} ; L^{2}(G)\right)} \leq\left\|p_{j}(v)\right\|_{L^{2}\left(\tau, T_{\lambda} ; L^{2}(G)\right)}, \quad \forall p_{j} \in \partial_{j} V\left(t ; S \mathscr{E}\left(\left.v\right|_{G}\right)\right) .
$$

In particular, we have

$$
\left\|\hat{p}_{j}\right\|_{L^{2}\left(\tau, T_{\lambda} ; L^{2}(G)\right)} \leq\left\|p_{j}(v)\right\|_{L^{2}\left(\tau, T_{\lambda} ; L^{2}(G)\right)}, \quad \forall p_{j}(v) \in \partial_{j} V\left(t ; S \mathscr{E}\left(\left.v\right|_{G}\right)\right) .
$$


Thus, $\hat{p}_{j}$ is the unique element of the closed convex set $\partial_{j} V\left(t ; S \mathscr{E}\left(\left.v\right|_{G}\right)\right)$ with minimal $L^{2}\left(\tau, T_{\lambda} ; L^{2}(G)\right)$-norm.

By definition, we have

$$
k_{j}^{*}\left(\mathscr{E}\left(p_{j}\left(v^{n}\right)\right)\right)=\left(\mathscr{E}\left(p_{j}\left(v^{n}\right)\right), q_{j}\left(v^{n}\right)\right)_{L^{2}\left(\tau, T_{\lambda} ; H\right)}
$$

for some $q_{j}\left(v^{n}\right) \in \mathscr{2}_{j}$. Moreover,

$$
q_{j}\left(v^{n}\right) \in \partial k_{j}^{*}\left(\mathscr{E}\left(p_{j}\left(v^{n}\right)\right)\right) .
$$

Since $\mathscr{E}$ is a bounded mapping of $L^{2}(G)$ into $H$, its adjoint is also a bounded mapping of $H$ into $L^{2}(G)$. With $2_{j}$ being time-independent, it follows from the definition of the set $2_{j}$ and from the Sobolev imbedding theorem that

$$
\mathscr{E}^{*}\left(q_{j}\left(v^{n}\right)\right) \longrightarrow \mathscr{E}^{*}\left(q_{j}\right) \quad \text { in } L^{2}\left(\tau, T_{\lambda} ; L^{2}(G)\right) .
$$

We have

$$
k_{j}^{*}\left(\mathscr{E}\left(\hat{p}_{j}\right)\right)-k_{j}^{*}\left(\mathscr{E}\left(p_{j}\left(v^{n}\right)\right)\right) \geq \int_{\tau}^{T_{\lambda}}\left(q_{j}\left(v^{n}\right), \mathscr{E}\left(\hat{p}_{j}\right)-\mathscr{E}\left(p_{j}\left(v^{n}\right)\right)\right) d t .
$$

It follows that

$$
q_{j} \in \partial k_{j}^{*}\left(\mathscr{E}\left(\hat{p}_{j}\right)\right)
$$

From (4.18) we get

$$
k_{j}^{*}\left(\mathscr{E}\left(p_{j}\left(v^{n}\right)\right)\right) \longrightarrow\left(\mathscr{E}\left(\hat{p}_{j}\right), q_{j}\right)_{L^{2}\left(\tau, T_{\lambda} ; H\right)}=k_{j}^{*}\left(\mathscr{E}\left(\hat{p}_{j}\right)\right) .
$$

An argument as done for $\hat{p}_{j}$ shows that $q_{j}$ is the unique element of the closed convex set

$$
k_{j}^{*}\left(\mathscr{E}\left(\hat{p}_{j}\right)\right)=\left(\mathscr{E}\left(\hat{p}_{j}, q_{j}\right)\right)_{L^{2}\left(\tau, T_{\lambda} ; H\right)}
$$

with minimal $L^{2}\left(\tau, T_{\lambda} ; H\right)$-norm.

Now we have $A(v)=\left.u\right|_{G}$ and therefore the nonlinear operator $A$ of the compact convex set $\mathscr{B}_{\tilde{C}}$ of $L^{2}\left(\tau, T_{\lambda} ; L^{2}(G)\right)$ into $\mathscr{B}_{\tilde{C}}$ is continuous. Applying the Schauder fixed point theorem, we deduce the existence of $u$, solution of (4.1).

The theorem is proved.

The main result of the paper is the following theorem.

Theorem 4.2. Suppose that all the hypotheses of Theorem 3.2 are satisfied. Let $\tilde{u}$ be a solution of (4.1) given by Theorem 4.1. Then

$$
V(\tau, \omega)=\sum_{j=1}^{2} \int_{\tau}^{T_{\lambda}} \int_{\Gamma}\left|\tilde{u}_{j}-\chi_{j}\right|^{2} d \sigma d t .
$$


398 An inverse problem for a nonlinear Schrödinger equation

Proof. Let $\tilde{u}$ be as in Theorem 4.1 and consider the Cauchy problem

$$
\begin{aligned}
\imath u^{\prime}+\Delta u+\lambda|u|^{2} u & =-\imath q u & & \text { in } \mathbb{R}^{2} \times\left(t, T_{\lambda}\right), \\
u(t) & =\tilde{u}(\cdot, t) & & \text { in } \mathbb{R}^{2} .
\end{aligned}
$$

With $\tilde{u}$ as in Theorem 4.1, then for a given $q \in 2$, there exists a unique solution $u$ of (4.26). Consider

$$
V(t ; \tilde{u}(t))=\inf \left\{\sum_{j=1}^{2} \int_{t}^{T} \int_{\Gamma}\left|u-\chi_{j}\right|^{2} d \sigma d t \forall q_{j} \in \mathscr{2}_{j}\right\} .
$$

Using a minimizing sequence, we get by a trivial argument

$$
V(t ; \tilde{u}(t))=\sum_{j=1}^{2} \int_{t}^{T_{\lambda}} \int_{\Gamma}\left|\hat{u}_{j}-\chi_{j}\right|^{2} d \sigma d s
$$

for some $\{\hat{u}, \hat{q}\}$, solution of (4.26).

From the dynamic programming principle, we deduce that

$$
V(t+h ; \hat{u}(t+h))=\sum_{j=1}^{2} \int_{t+h}^{T_{\lambda}} \int_{\Gamma}\left|\hat{u}_{j}-\chi_{j}\right|^{2} d \sigma d s .
$$

Hence

$$
\frac{d}{d t} V(t ; \hat{u}(t))=-\sum_{j=1}^{2} \int_{\Gamma}\left|\hat{u}_{j}(t, \cdot)-\chi_{j}\right|^{2} d \sigma
$$

Since $\tilde{u}(\cdot, t)=\hat{u}(\cdot, t)$ in $\mathbb{R}^{2}$, we get

$$
\frac{d}{d t} V(t ; \hat{u}(t))=-\sum_{j=1}^{2} \int_{\Gamma}\left|\tilde{u}(\cdot, t)-\chi_{j}(\cdot, t)\right|^{2} d \sigma .
$$

It follows that

$$
\begin{aligned}
V\left(T_{\lambda} ; \tilde{u}\left(T_{\lambda}\right)\right)-V(\tau ; \omega) & =-V(\tau ; \omega) \\
& =-\sum_{j=1}^{2} \int_{\tau}^{T_{\lambda}} \int_{\Gamma}\left|\tilde{u}_{j}(\cdot, t)-\chi_{j}(\cdot, t)\right|^{2} d \sigma d s
\end{aligned}
$$

The theorem is proved.

Remark 4.3. The general case when the cost function depends also on the control is open. 


\section{References}

[1] S. A. Avdonin, S. Lenhart, and V. Protopopescu, Solving the dynamical inverse problem for the Schrödinger equation by the boundary control method, Inverse Problems $\mathbf{1 8}$ (2002), no. 2, 349-362.

[2] V. Barbu, Necessary conditions for distributed control problems governed by parabolic variational inequalities, SIAM J. Control Optim. 19 (1981), no. 1, 64-86.

[3] G. Fibich, Self-focusing in the damped nonlinear Schrödinger equation, SIAM J. Appl. Math. 61 (2001), no. 5, 1680-1705.

[4] J. Ginibre and G. Velo, On a class of nonlinear Schrödinger equations. I. The Cauchy problem, general case, J. Funct. Anal. 32 (1979), no. 1, 1-32.

[5] T. Kato, On nonlinear Schrödinger equations, Ann. Inst. H. Poincaré Phys. Théor. 46 (1987), no. 1, 113-129.

[6] M. J. Landman, G. C. Papanicolaou, C. Sulem, and P.-L. Sulem, Rate of blowup for solutions of the nonlinear Schrödinger equation at critical dimension, Phys. Rev. A (3) 38 (1988), no. 8, 3837-3843.

[7] M. J. Landman, G. C. Papanicolaou, C. Sulem, P.-L. Sulem, and X. P. Wang, Stability of isotropic singularities for the nonlinear Schrödinger equation, Phys. D 47 (1991), no. 3, 393-415.

[8] B. J. LeMesurier, G. C. Papanicolaou, C. Sulem, and P.-L. Sulem, The focusing singularity of the nonlinear Schrödinger equation, Directions in Partial Differential Equations (Madison, Wis, 1985), Publ. Math. Res. Center Univ. Wisconsin, vol. 54, Academic Press, Massachusetts, 1987, pp. 159-201.

[9] L Local structure of the self-focusing singularity of the nonlinear Schrödinger equation, Phys. D 32 (1988), no. 2, 210-226.

[10] C. Popa, Feedback laws for nonlinear distributed control problems via Trotter-type product formulae, SIAM J. Control Optim. 33 (1995), no. 4, 971-999.

[11] The relationship between the maximum principle and dynamic programming for the control of parabolic variational inequalities, SIAM J. Control Optim. 35 (1997), no. 5, 1711-1738.

[12] B. A. Ton, An inverse problem for the Schrödinger equation, submitted to Inverse Problems.

Bui An Ton: Department of Mathematics, University of British Columbia, VANCouver, BC, CANada V6T 1Z2

E-mail address: bui@math.ubc.ca 


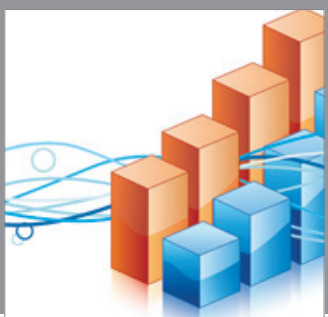

Advances in

Operations Research

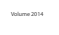

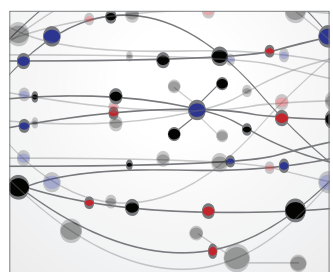

\section{The Scientific} World Journal
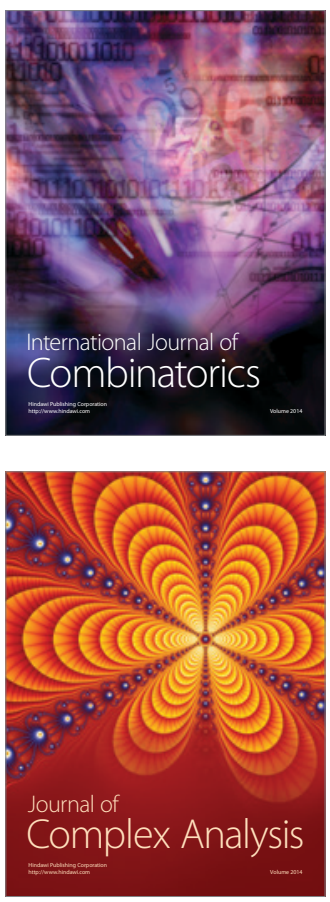

International Journal of

Mathematics and

Mathematical

Sciences
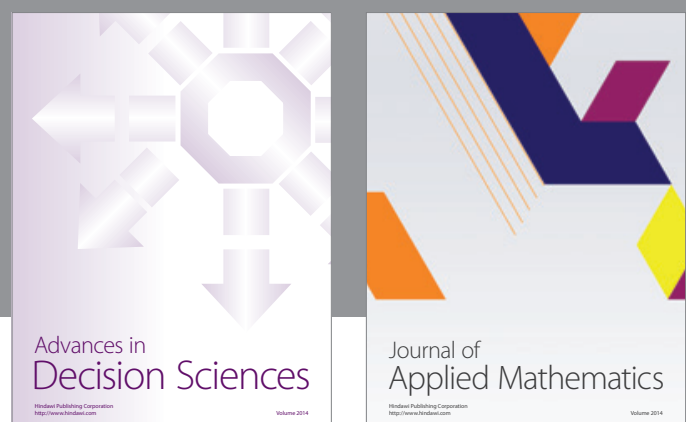

Journal of

Applied Mathematics
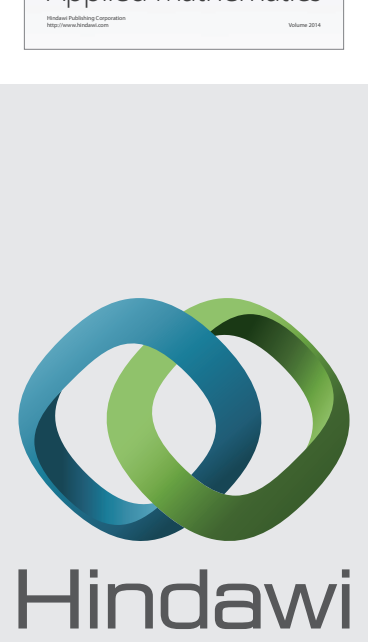

Submit your manuscripts at http://www.hindawi.com
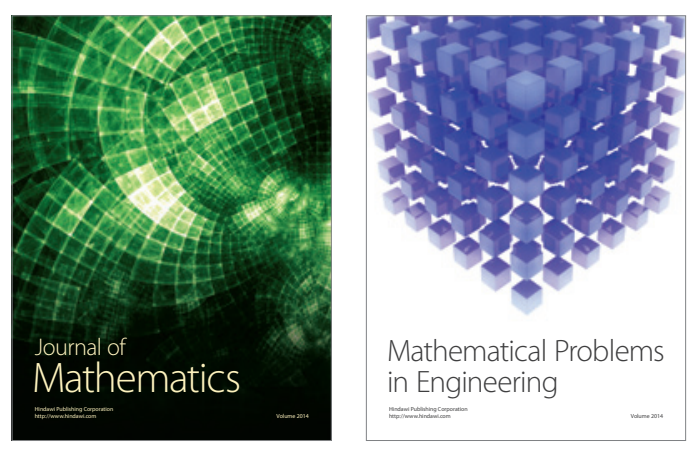

Mathematical Problems in Engineering
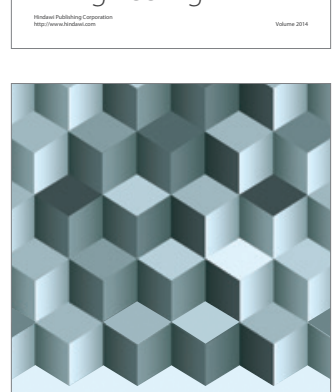

Journal of

Function Spaces
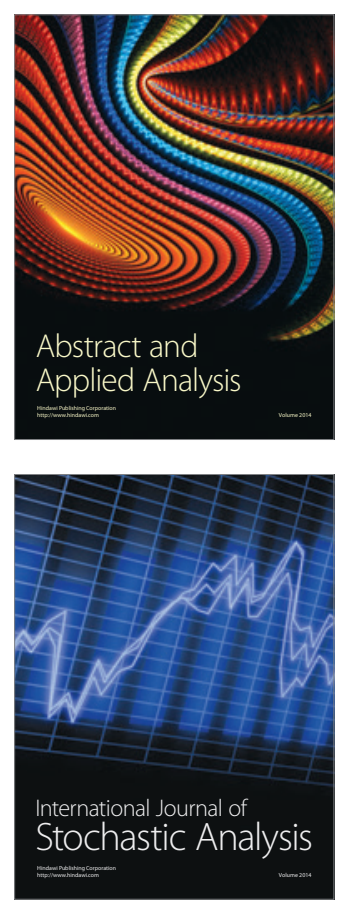

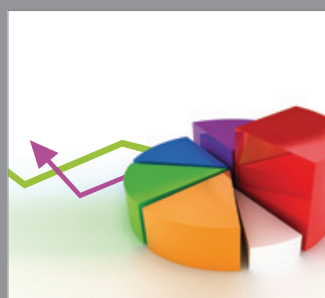

ournal of

Probability and Statistics

Promensencen
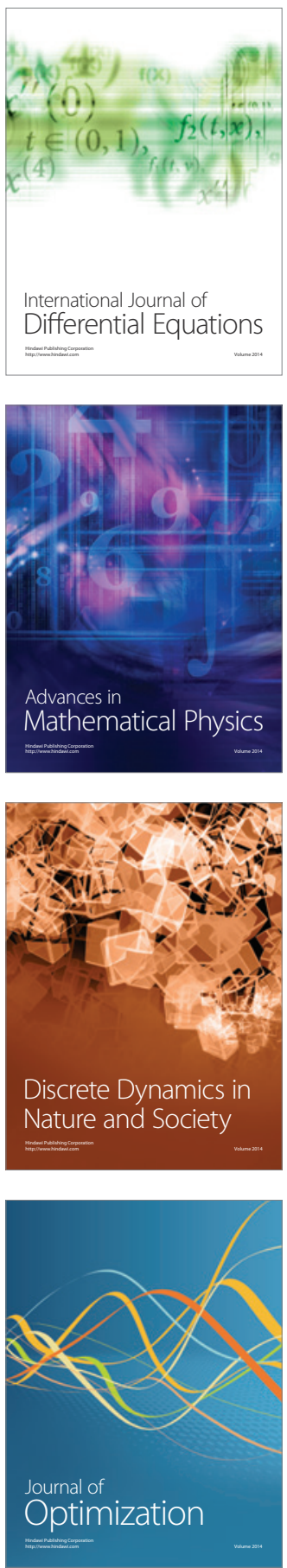